\title{
Massive variable stars at very low metallicity?
}

\author{
Dominik J. Bomans and Kerstin Weis \\ Astronomical Institute, Ruhr-University Bochum, \\ Universitätsstr. 150, 44801, Bochum, Germany \\ email: bomans@astro.rub.de, kweis@astro.rub.de
}

\begin{abstract}
Observational contraints on the evolution and instabilities of massive stars at very low metallicities are limited. Most of the information come from HST observations of one target, I Z $\mathrm{w}$ 18. Recent distance estimates of I $\mathrm{Zw}_{\mathrm{w}} 18$ put it at $17 \mathrm{Mpc}$, moving detailed studies of single stars clearly beyond the range of current ground based telescopes. Since massive stars with metallcities of $1 / 10$ of solar and below are our best proxies for massive stars in (proto-) galaxies around the time of reionization, finding them and studying their evolution and instabilities is of premium importance for our understanding of galaxy formation, feedback, and the IGM reionization. Here we present pilot study results of variable stars in two more nearby extremely low metallicity galaxies, UGC 5340 and UGCA 292, and comment on the possibilities of more detailed studies of variable massive stars with new ground-based instrumentation.
\end{abstract}

Keywords. stars: variables: other, stars: evolution, stars: early-type, (stars:) supergiants, stars: winds, outflows, galaxies: stellar content, galaxies: irregular, instrumentation: spectrographs

\section{Why is very low metallicity exciting?}

It is long known from theory of stellar evolution and stellar atmospheres that there are significant changes when going to stars at low metallicity. These effects are already apparent in the Magellanic Clouds, which provide nice laboratories for massive stars at 0.4 and 0.2 of solar metallicity. Still, there is a significant gap between the metallicity of the Magellanic Clouds and the metallicities observed at high redshifts, despite a significant spread of metallicities observed in DLA and Lyman break galaxies. This is especially true for the epoch of galaxy formation to the end of reionization $(z>6)$ (e.g., Schaerer \& de Barros 2010). Observational studies of massive stars at metallicities significantly below $1 / 10$ of solar are therefore important for our understanding not only of galaxy formation but also stellar feedback at high redshift. Massive stars and subsequent supernovae provide the energy input driving galactic outflows and winds (Leitherer et al. 1992), which are critical ingredients of galaxy formation and evolution. At very low metallicity, stellar evolution, stellar atmospheres, and the instabilities in stars should be very different. This affects stellar winds, late evolutionary phases, variability, (evolution of) rotation, convection, SN types, binary star evolution, and progenitors of long GRBs.

\section{Nearby very low metallicity galaxies}

To get a better handle on the stellar population and their time dependent energy input to their host galaxies in the early universe, observations of the best possible local proxies are mandatory. Unfortunately, such extreme metal-poor galaxies (metallicities of less than $\sim 1 / 10$ of solar) are quite rare objects (e.g., Kunth \& Östlin 2000). The prototype of these galaxies is I Zw 18. Not surprisingly, it was repeatably observed with HST, but it turned out to be quite difficult to study due to high crowding, high and variable background and 


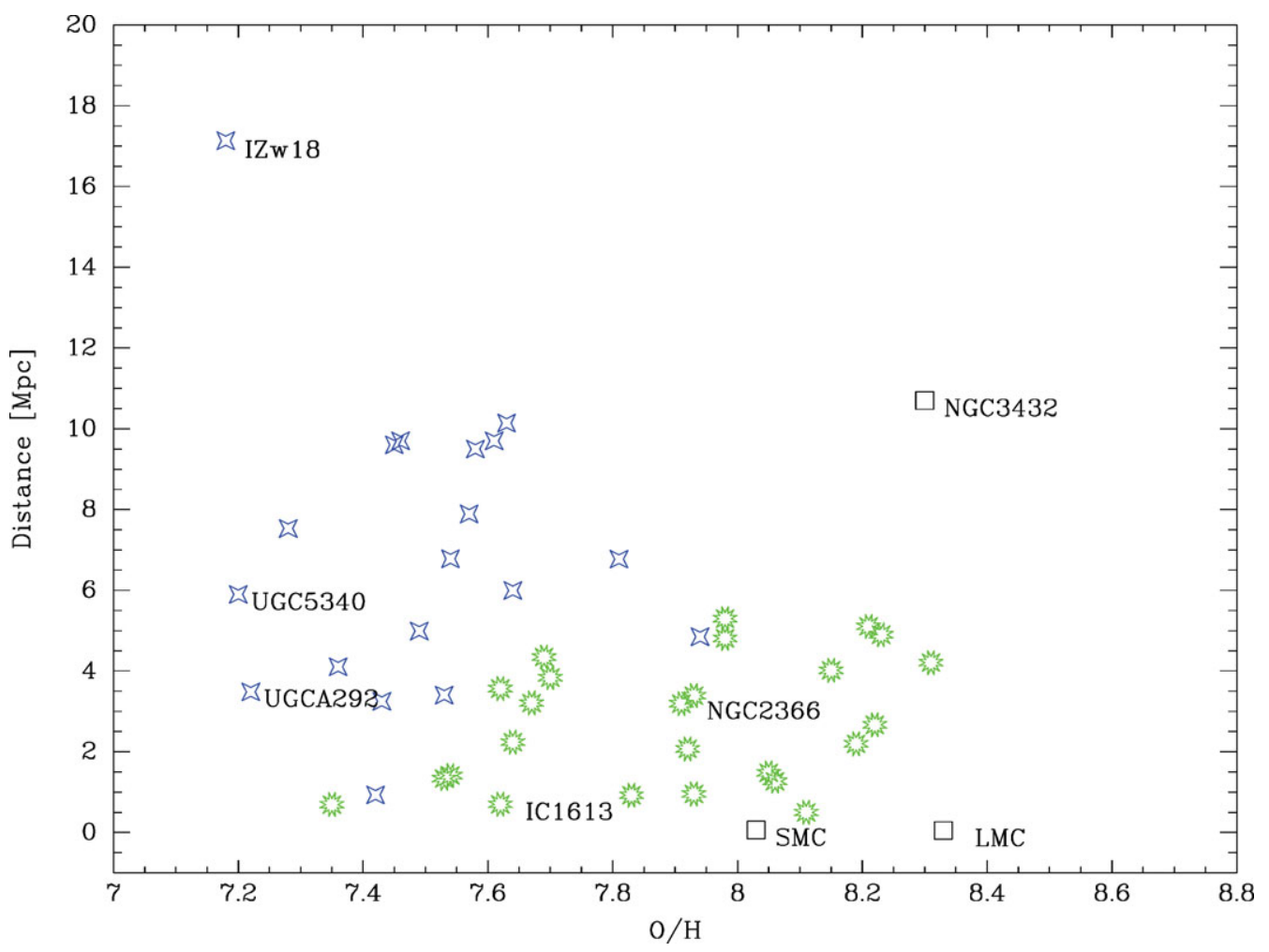

Figure 1. Distance versus metallicity of a compilation of the most metal poor galaxies in the local universe. Green stars are from the compilation of Lee et al. (2006), black squares some important other objects, blue stars are all currently known nearby very low metallicity galaxies.

faintness of its stars. I Zw 18 has now a securely determined distance of $18 \mathrm{Mpc}$ (Aloisi et al. 2007; Fiorentini et al. 2010), which places it beyond the reach for ground based studies of its stars. The analysis of the HST data material showed that I Zw 18 does contain five periodic variables, 3 of them inside the Cepheid instability strip (2 of the Cepheids have periods $>100$ days), and 34 candidat/non-periodic variables (Fiorentino et al. 2010). Since the Meynet \& Maeder (2005) rotation models imply an extension of the mass range of stars with LBV-like instabilities downward (e.g., Weis 2010, these proceedings), the location of the brightest and bluest of these candidate variables in I $\mathrm{Zw} 18$ is consistent with the CMD location of rapidly rotating LBV-like stars.

Clearly, for detailed studies of massive stars at these metallicities, more targets at lower distance are important. The basic problem to reach this aim is that such low metallicities are found locally only in low mass galaxies (e.g. Lee et al. 2006). Therefore a significant recent star formation rate in these galaxies is needed to ensure useful sample sizes of (very) massive and therefore short lived stars. The short evolutionary time scales therefore implies that not every low metallicity dwarf galaxy will provide examples of all short lived, transitional phases.

\section{Very low metallicity galaxies in the local volume}

Which are the galaxies with significant recent star formation at low metallcity and how many do exist in the Local Volume? As Local Volume we define here the sphere with $11 \mathrm{Mpc}$ radius of the Milky Way. It is roughly the maximal volume in which massive 
single stars are accessible for detailed spectral analysis with $10 \mathrm{~m}$ class ground based telescopes, e.g. Bresolin et al. (2001). The problem for the study of single stars out to these distances is not only the faintness of the targets but also the spatial resolution. Dense groups and clusters of massive stars become unresolved, or just barely resolved, producing a significant light contamination to targets in or near them. In the case of luminous transients, like LBV outbursts, these problems are significantly reduced, as the star outshines its environment. In such a case the Local Volume may be even a conservative limit, see for example the LBV transient in NGC 3432 (Pastorello et al. 2010). In the case of very bright transients, time enters the problem. Due to their transient nature, they will unpredictively be bright enough for study. The other problem is that studying only these transient introduces a classical Malmquist bias to a lot of the analyses. We started to compile a data base of very low metallicity local galaxies, the current state of it is plotted in Fig.1. While I Zw 18 is clearly not the best target anymore, it is doable with the HST, and several more potentially useful targets within $8 \mathrm{Mpc}$ are apparent.

\section{Apparently very massive variable stars a low metallicity}

UGC 5340 is an extremely low metallicity galaxy with an abundance of $\sim 1 / 40$ solar. The LBV candidate in UGC 5340 was a serendipitous spectroscopic discovery. When taking a spectrum of a HII knot in 2008, Pustilnik et al. (2008) noted that it is significantly different from a spectrum taken 3 years earlier. The difference spectrum clearly showed $\mathrm{P}$ Cygni profiles in the Balmer lines and a blue continuum. They interpreted this finding as the brightening of an LBV, an interpretation later supported by Izotov \& Thuan (2009).

Still, one should still be skeptical, since slight misalignment between the slit position of the observations in such a relatively distant $(\mathrm{D} \sim 8 \mathrm{Mpc})$ and a complex background may lead to spurious results. To check the presence of a variable source, we compiled a ground based light curve of the object using own and archival imaging data. The result is plotted in Fig.2. Clearly, the knot is variable by more that a 1 mag over the last 50 years. If the measurement from 1988 defines the quiescent state of the most luminous star in the knot, than the star showed brightening by $>2^{\text {mag }}$ since then. For the interpretation one has to keep in mind that these measurements are integrated values for the unresolved (or barely resolved) ionizing cluster of an HII region. Therefore the age of the cluster should be below $3 \times 10^{6} \mathrm{yr}$. Our preliminary STARBURST99 simulations even imply $\sim 1 \times 10^{6} \mathrm{yr}$ (Bomans et al. 2010).

All these pieces of evidence seem to be consistent with a very massive, highly variable star, dominating the luminosity and color of the cluster in its bright phases. Before jumping to the interpretation of an LBV, there are a few odd aspects: the brightening appears to be at constant or bluer color, which excludes a classical S Dor variability (e.g. van Genderen 2001). The wind terminal velocity estimated from the 2008 difference spectrum is $\sim 800 \mathrm{~km} \mathrm{~s}^{-1}$, which looks more more like a wind than mass ejection event (a giant eruption).

NGC 2366 is a strongly star forming (Lee et al. 2009) dwarf irregular galaxy at a distance of $3 \mathrm{Mpc}$ and a metallicity of $\sim 1 / 10$ solar. The massive stars are clearly driving material out into the halo of this galaxy (Martin 1998; van Eymeren et al. 2009), making it a good laboratory for stellar feedback studies. In NGC 2366, Drissen et al. (2001) noted the sudden appearance of a stellar source inside its brightest giant His region. They found an increase by $3.1^{\text {mag }}$ to $\mathrm{V} \sim-10.2^{\text {mag }}$ and a rise in brightness while getting hotter (bluer colors). The spectra changed with time (Petit et al. 2006) and the early spectra are similar 

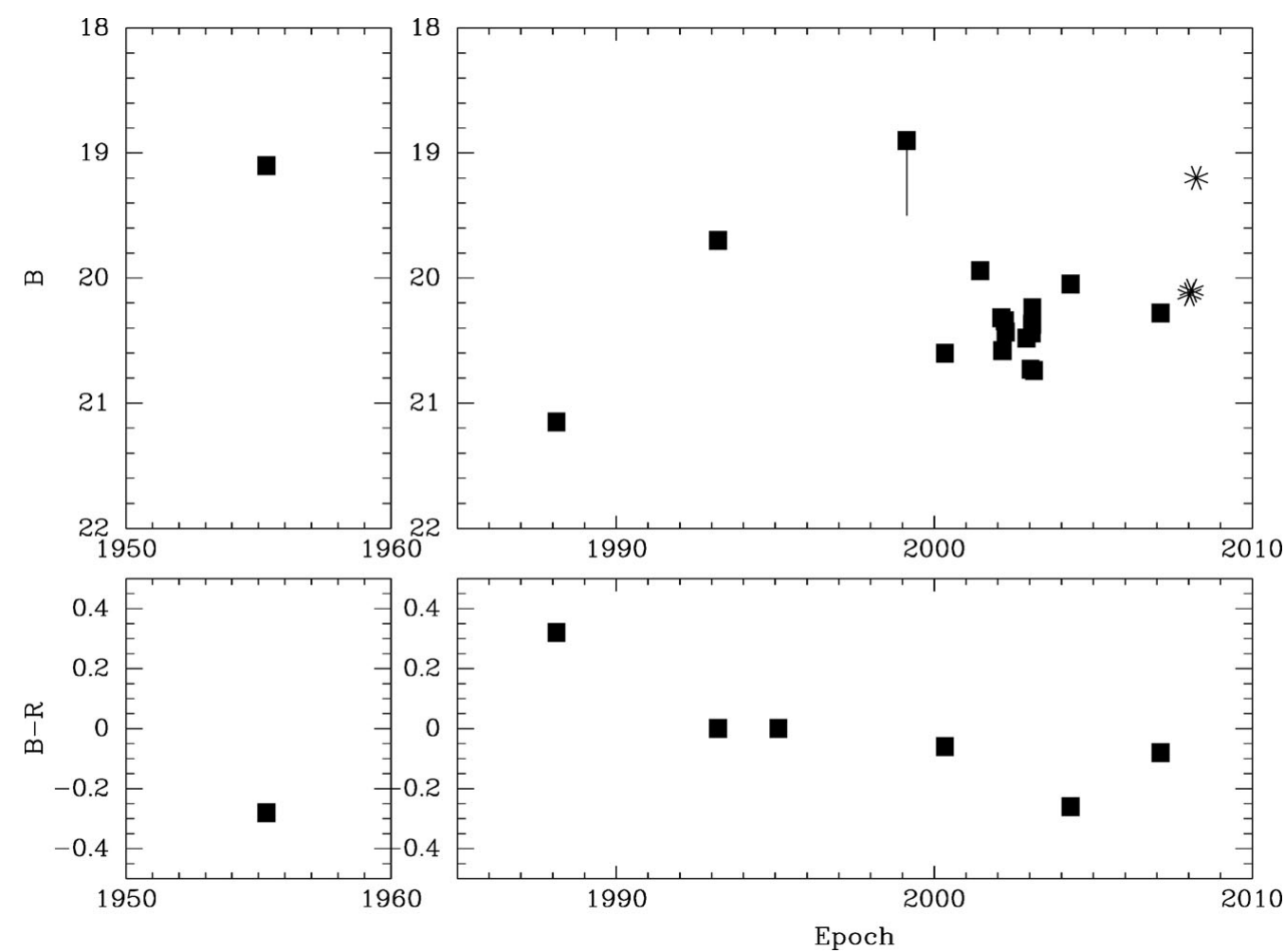

Figure 2. Lightcurve of the cluster plus transient source in UGC 5340. Black squares are archival and our photometric data (mostly from CCD), asterisks denote spectroscopic fluxes converted to $\mathrm{R}$ band magnitudes.

to the one of the UGC 5340 transient. Again the spectra are more like wind instead of sudden mass ejection (giant eruption) and the wind terminal velocity $\sim 250 \mathrm{~km} \mathrm{~s}^{-1}$.

There appear to be distinct similarities between the UGC 5340 transient and NGC 2366 V1. One is tempted to speculate that we see a LBV-like variability, but somewhat different from classical S Dor cycle. Within that cycle the star should encounter the bistability jump, which is function of metallicity. This might lead to a less pronounced change in the star's spectrum (and the S Dor variability) and may yield a different spectroscopic behaviour for LBVs like UGC 5340 and NGC 2366 V1 (see e.g., Weis 2010, these proceedings).

\section{Very low metallicity variables at lower mass?}

Observational results on the evolution of massive (but not extremely massive) stars and the variability at very low metallicities are very sparse, too. The I Zw 18 results of Fiorentino et al. (2010) show a rich population of possible variables especially in the blue loop area of lower mass supergiants. Differences to higher metallicity samples are not yet clear, since the apparent statistics needs to take the complex star formation history in I Zw 18 into account.

V39 in IC 1613, a very metallicity dwarf irregular galaxy in the Local Group (1/14 of solar), is another example of strange variable stars at the lower end of the mass region expected for LBV-like stars (Herrero et al. 2010). 


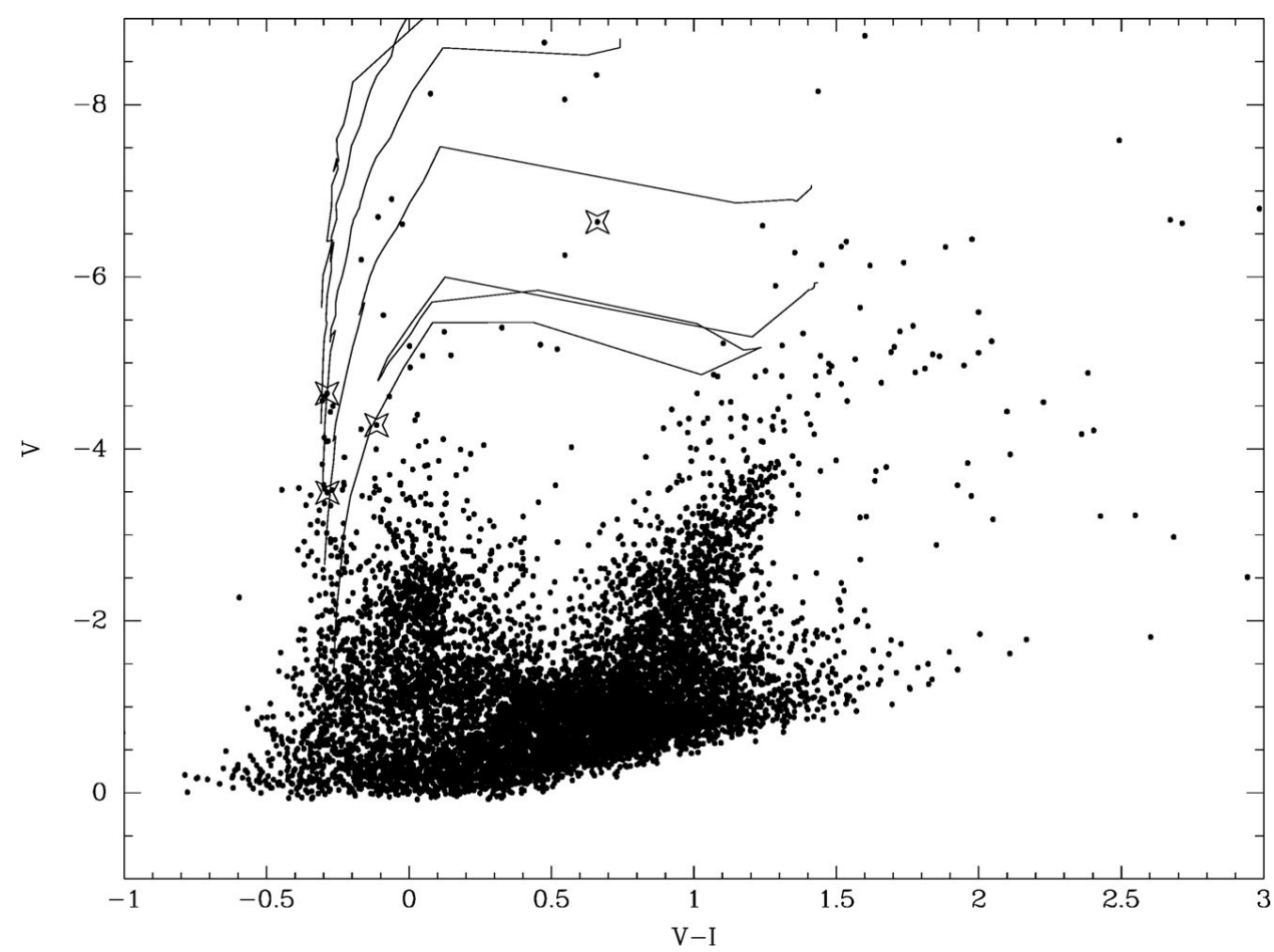

Figure 3. Preliminary HST CMD of UGCA 292. The 4 objects marked with stars are high probability variable stars. Geneva tracks (without rotation for $60,40,25,15$, and $9 \mathrm{M}_{\odot}$ are overplotted.

Using our database of very low metallicity galaxies, we startet a pilot project to search for massive variable stars in these galaxies using HST archival data. Fig.3 shows as an example the color-magnitude diagram (CMD) of UGCA 290 ( 1/40 of solar metallicity). The HST data provided a high quality CMD with clear structure in the region of upper main sequence and blue supergiant plume. We detected four high probability variables, one of which is located in the Hertzsprung gap. This pilot analysis based on only two epochs can can obviously provide just a snapshot of the variable massive star population. Photometric monitoring will be the next step, where the HST data provide useful priors for the photometry from ground-based data.

\section{Instrumentation aspects}

Imaging and photometric monitoring are important for the identification and characterization of the massive (variable) stars, but for many parameters, at least intermediate dispersion spectroscopy is needed. Together with our need for high spatial resolution this defines a mayor challenge, which at least two new instruments at $8-10 \mathrm{~m}$ class telescope will help to overcome. At the LBT, running since 2010, the LUCIFER NIR multi-object spectrograph, is fully utilizing the very good median seeing in the NIR at Mt. Graham. At the VLT, starting 2012, the multi-IFU spectrograph MUSE with adaptive optics will be big progress for solving the crowding problem. Both instruments provide good spectral resolution and high throughput for the spectral analysis of stellar parameters in Local Volume galaxies. Clearly, the future ELTs will allow the study of massive stars in 
galaxies well beyond this distance (e.g. Evans 2010, these proceedings), but there are several objects with can be studied with current instrumentation.

\section{Conclusions}

Stars are very different at $\sim 1 / 40$ solar metallicity than SMC stars, as recently shown by comparing their new HST-COS spectrum of the I Zw 18 cluster with UV spectra of SMC (1/5 solar metallicity) stars (Heap et al. 2010). The metal lines is the COS spectrum were extremely weak, even compared to SMC stars. Still, 'normal' stellar types exist, even Cepheids and WR stars. Very low metallicity galaxies are rare in the Local Volume, but there are a few good targets. Unfortunately, I Zw 18 is not necessarily one of them.

In UGC 5340 a LBV-like transient appeared in 2008. It appears to have similar properties as NGC 2366 V1. Both do not behave like normal, high metallicity LBVs, therefore we may see the effects of metallicity on the underlying instability. IC $1613 \mathrm{~V} 39$, the variable stars detected in UGCA 290, and the bright, blue non-periodic variable in I Zw 18 point at roads to the physics of very metal-poor massive stars.

New instruments are providing access to spectroscopy of the very metal poor active OB stars NOW!

\section{References}

Aloisi, A., Clementini, G., Tosi, M., Annibali, F. et al. 2007, ApJ (Letters), 667, L151

Bomans, D.J., et al. 2010, in: P. Williams \& G. Rauw (eds.), The multi-wavelength view of Hot, Massive Stars, $39^{\text {th }}$ Liège International Astrophysical Colloquium, in press

Bresolin, F., Kudritzki, R.-P., Mendez, R. H. \& Przybilla, N. 2001, ApJ (Letters), 548, L159

Drissen, L., Crowther, P. A., Smith, L. J., Robert, C. et al. 2001, ApJ, 546, 484

van Eymeren, J., Marcelin, M., Koribalski, B., Dettmar, R.-J. et al. 2009, A\& A, 493, 511

Fiorentino, G., Contreras Ramos, R., Clementini, G., Marconi, M. et al. 2010, ApJ, 711, 808

van Genderen, A. M. 2001, $A \& \mathcal{E} A, 366,508$

Heap, S. et al. 2010, in: P. Williams \& G. Rauw (eds.), The multi-wavelength view of Hot, Massive Stars, $39^{\text {th }}$ Liège International Astrophysical Colloquium, in press

Herrero, A., Garcia, M., Uytterhoeven, K., Najarro, F. et al. 2010, A\&\&A, 513, A70

Izotov, Y. I. \& Thuan, T. X. 2009, ApJ, 690, 1797

Kunth, D. \& Östlin, G. 2000, A\&AR, 10, 1

Lee, H., Skillman, E. D., Cannon, J. M., Jackson, D. C. et al. 2006, ApJ, 647, 970

Lee, J. C., Kennicutt, R. C., José G. Funes, S. J., Sakai, S. et al. 2009, ApJ, 692, 1305

Leitherer, C., Robert, C. \& Drissen, L. 1992, ApJ, 401, 596

Martin, C. L. 1998, ApJ, 506, 222

Meynet, G. \& Maeder, A. 2005, A\&SA, 429, 581

Pastorello, A., Botticella, M. T., Trundle, C., Taubenberger, S. et al. 2010, MNRAS 408, 181

Petit, V., Drissen, L., \& Crowther, P. A. 2006, AJ, 132, 1756

Pustilnik, S. A., Tepliakova, A. L., Kniazev, A. Y., \& Burenkov, A. N. 2008, MNRAS, 388, L24

Schaerer, D. \& de Barros, S. 2010, A\&3A, 515, A73 